PROCEEDINGS OF THE

AMERICAN MATHEMATICAL SOCIETY

Volume 126, Number 6, June 1998, Pages 1811-1819

S 0002-9939(98)04254-3

\title{
A MAXIMAL INEQUALITY FOR PARTIAL SUMS OF FINITE EXCHANGEABLE SEQUENCES OF RANDOM VARIABLES
}

\author{
ALEXANDER R. PRUSS
}

(Communicated by Stanley Sawyer)

\begin{abstract}
Let $X_{1}, X_{2}, \ldots, X_{2 n}$ be a finite exchangeable sequence of Banach space valued random variables, i.e., a sequence such that all joint distributions are invariant under permutations of the variables. We prove that there is an absolute constant $c$ such that if $S_{j}=\sum_{i=1}^{j} X_{i}$, then

$$
P\left(\sup _{1 \leq j \leq 2 n}\left\|S_{j}\right\|>\lambda\right) \leq c P\left(\left\|S_{n}\right\|>\lambda / c\right),
$$

for all $\lambda \geq 0$. This generalizes an inequality of Montgomery-Smith and Latała for independent and identically distributed random variables. Our maximal inequality is apparently new even if $X_{1}, X_{2}, \ldots$ is an infinite exchangeable sequence of random variables. As a corollary of our result, we obtain a comparison inequality for tail probabilities of sums of arbitrary random variables over random subsets of the indices.
\end{abstract}

Montgomery-Smith [8] and Latała [7] have independently proved that if $X_{1}, \ldots$, $X_{n}$ are independent and identically distributed Banach space valued random variables, then

$$
P\left(\sup _{1 \leq j \leq k}\left\|\sum_{i=1}^{j} X_{i}\right\|>\lambda\right) \leq c P\left(\left\|\sum_{i=1}^{k} X_{i}\right\|>\lambda / c\right),
$$

whenever $\lambda \geq 0$ and $1 \leq k \leq n$, where $c$ is an absolute constant. It is obvious that this cannot hold for arbitrary independent random variables; as MontgomerySmith [8] notes, we need only let $k=n=2, X_{1} \equiv 1$ and $X_{2} \equiv-1$ to see this. Lévy's inequality says that (1) also holds for arbitrary independent symmetric random variables $X_{i}$ (not necessarily identically distributed). For positive random variables, (1) is trivial, of course.

A natural and much-studied extension of the concept of independent and identically distributed random variables is that of exchangeable random variables. We say that a finite sequence $X_{1}, \ldots, X_{n}$ of (not necessarily independent) random variables is exchangeable if the $n$-tuples $\left(X_{1}, \ldots, X_{n}\right)$ and $\left(X_{\pi(1)}, \ldots, X_{\pi(n)}\right)$ both have the same distribution whenever $\pi$ is a permutation of $[n] \stackrel{\text { def }}{=}\{1, \ldots, n\}$. Evidently an exchangeable sequence of independent random variables is precisely a sequence of independent and identically distributed random variables.

Received by the editors August 2, 1996 and, in revised form, December 2, 1996.

1991 Mathematics Subject Classification. Primary 60E15.

Key words and phrases. Sums of exchangeable random variables, maximal inequalities.

(C) 1998 American Mathematical Society 
Note that given any sequence $Y_{1}, \ldots, Y_{n}$ of random variables (with no independence or identical distribution assumptions), if $\tau$ is a random permutation of $[n]$ (with all $n$ ! such permutations being equally likely, and with $\tau$ independent of the $\left.Y_{i}\right)$, and if we put $X_{i}=Y_{\tau(i)}$, then $X_{1}, \ldots, X_{n}$ is an exchangeable sequence. If the $Y_{i}$ are constants, then this is just sampling without replacement from an urn, and moreover, in this case unless the $Y_{i}$ are all the same, the sequence $X_{1}, \ldots, X_{n}$ cannot be extended to an exchangeable sequence of length $n+1$ (i.e., there is no exchangeable sequence $X_{1}^{\prime}, \ldots, X_{n}^{\prime}, X_{n+1}^{\prime}$ such that the $n$-dimensional distribution of $\left(X_{i}\right)_{1 \leq i \leq n}$ coincides with that of $\left.\left(X_{i}^{\prime}\right)_{1 \leq i \leq n}\right)$.

An infinite sequence of random variables is said to be exchangeable if every finite subsequence is exchangeable. De Finetti [2] has proved that the probability distribution of an infinite exchangeable sequence of random variables is a mixture of probability distributions of independent and identically distributed sequences. This, together with Montgomery-Smith and Latała's inequality (1), implies that

$$
P\left(\left\|\sum_{i=1}^{k} X_{i}\right\|>\lambda\right) \leq c P\left(\left\|\sum_{i=1}^{n} X_{i}\right\|>\lambda / c\right),
$$

for $1 \leq k \leq n$, providing that $X_{1}, \ldots, X_{n}$ can be extended to an infinite sequence of exchangeable random variables. (The reader may wish to see, e.g., $[1,3,4,5,9,10]$ for work on the question of extending finite exchangeable sequences to infinite ones or at least to longer finite ones.)

However, (2) need not hold if $X_{1}, \ldots, X_{n}$ are assumed to be merely exchangeable. For, let $V_{1}, \ldots, V_{n}$ be any sequence of vectors, not all zero, such that $V_{1}+\cdots+V_{n}=$ 0 . Let $\tau$ be a random permutation of $[n]$, with all $n$ ! such permutations being equally likely. Let $X_{i}=V_{\tau(i)}$ (this is just sampling the $V_{j}$ from an urn without replacement). It is clear that $X_{1}, \ldots, X_{n}$ are exchangeable. But, $X_{1}+\cdots+X_{n}=$ $V_{1}+\cdots+V_{n}=0$, while $P\left(\left\|X_{1}\right\|>0\right)>0$ since the $V_{i}$ are not all zero, so that (2) cannot hold if $k=1$ and $\lambda>0$ is sufficiently small.

Hence, we see that (2) must hold if $X_{1}, \ldots, X_{n}$ can be extended to an infinite sequence of exchangeable random variables, but need not hold if $X_{1}, \ldots, X_{n}$ are merely assumed to be exchangeable. Professor Stephen J. Montgomery-Smith has asked the author whether perhaps (2) would hold if $X_{1}, \ldots, X_{n}$ can be extended to an exchangeable sequence of random variables of length $2 n$. He also noted that

$$
\begin{aligned}
& P(\|X\|>\lambda)=P(\|X+Y+X+Z-Y-Z\|>2 \lambda) \\
& \quad \leq P\left(\|X+Y\|>\frac{2}{3} \lambda\right)+P\left(\|X+Z\|>\frac{2}{3} \lambda\right)+P\left(\|Y+Z\|>\frac{2}{3} \lambda\right) \\
& \quad=3 P\left(\|X+Y\|>\frac{2}{3} \lambda\right),
\end{aligned}
$$

whenever $X, Y, Z$ are exchangeable, which shows that the answer is positive for $n=2$.

We shall show that the answer to Professor Montgomery-Smith's question is positive in general, and in fact a length of $2 n$ is not needed, but any length at least as large as $\gamma n$ will do, where $\gamma$ is any fixed real number strictly greater than one; moreover we not only obtain (2), but also the maximal inequality (1).

Theorem 1. Fix $\gamma>1$. Let $n^{\prime}$ be an integer at least as large as $\gamma n$. Let $X_{1}, \ldots, X_{n^{\prime}}$ be an exchangeable sequence of random variables. Then,

$$
P\left(\sup _{1 \leq j \leq k}\left\|\sum_{i=1}^{j} X_{i}\right\|>\lambda\right) \leq c P\left(\left\|\sum_{i=1}^{k} X_{i}\right\|>\lambda / c\right),
$$


whenever $\lambda \geq 0$ and $1 \leq k \leq n$, where $c=c(\gamma) \in(0, \infty)$ is a constant depending only on $\gamma$.

This shows that (1) holds for $1 \leq k \leq n$ providing $X_{1}, \ldots, X_{n}$ can be extended to an exchangeable sequence of random variables of length at least $\gamma n$. The proof of Theorem 1 will be given later on in this paper.

We immediately obtain the following corollary which is apparently new in itself.

Corollary 1. Let $X_{1}, X_{2}, \ldots$ be an infinite exchangeable sequence of Banach valued random variables. Then, the Lévy-type maximal inequality (1) holds for all $k \geq 1$, where $c$ is an absolute constant.

We write $\lfloor x\rfloor$ for the greatest integer less than or equal to $x$. Put $\lceil x\rceil=-\lfloor-x\rfloor$. We then have the following maximal inequality.

Corollary 2. Fix $\rho \in(0,1)$. Let $X_{1}, \ldots, X_{n}$ be an exchangeable sequence of random variables with $\rho n \geq 1$. Let $m=\lfloor\rho n\rfloor$. Then,

$$
P\left(\sup _{1 \leq j \leq n}\left\|\sum_{i=1}^{j} X_{i}\right\|>\lambda\right) \leq c P\left(\left\|\sum_{i=1}^{m} X_{i}\right\|>\lambda / c\right),
$$

for all $\lambda \geq 0$, where $c=c(\rho) \in(0, \infty)$ is an absolute constant depending only on $\rho$.

In particular, if $X_{1}, \ldots, X_{2 n}$ are exchangeable, then

$$
P\left(\sup _{1 \leq j \leq 2 n}\left\|\sum_{i=1}^{j} X_{i}\right\|>\lambda\right) \leq c P\left(\left\|\sum_{i=1}^{n} X_{i}\right\|>\lambda / c\right)
$$

where $c=c\left(\frac{1}{2}\right)$ is an absolute constant.

Proof of Corollary 2. Set $S_{j}=\sum_{i=1}^{j} X_{i}$. Let $\gamma=1 / \rho$. Then, $n \geq \gamma m$. Hence,

$$
P\left(\sup _{1 \leq j \leq m}\left\|S_{j}\right\|>\lambda\right) \leq c P\left(\left\|S_{m}\right\|>\lambda / c\right),
$$

by Theorem 1 , where $c$ depends only on $\gamma=1 / \rho$.

Note that $n / m \leq 2 / \rho$ (this uses the assumption that $\rho n \geq 1$ ). Let $N=\lceil n / m\rceil$. Then, $N \leq 1+2 / \rho$. For $0 \leq k<N$, consider the event

$$
E_{k}=\left\{\sup _{1 \leq j \leq m}\left\|\sum_{i=m k+1}^{\min (m k+j, n)} X_{i}\right\|>\lambda / N\right\} .
$$

By exchangeability and (4),

$$
P\left(E_{k}\right)=P\left(\sup _{1 \leq j \leq \min (m(k+1), n)-(m k+1)}\left\|\sum_{i=1}^{j} X_{i}\right\|>\lambda\right) \leq c P\left(\left\|\sum_{i=1}^{m} X_{i}\right\|>\lambda / c\right) .
$$

But is easy to see that if $\omega \notin \bigcup_{k=0}^{N-1} E_{k}$, then $\left\|S_{j}(\omega)\right\| \leq \lambda$, so that

$$
\begin{aligned}
P\left(\sup _{1 \leq j \leq n}\left\|S_{j}\right\|>\lambda\right) & \leq P\left(\bigcup_{k=0}^{N-1} E_{k}\right) \leq N c P\left(\left\|S_{m}\right\|>\lambda / N c\right) \\
& \leq(1+2 / \rho) c P\left(\left\|S_{m}\right\|>\frac{\lambda}{(1+2 / \rho) c}\right)
\end{aligned}
$$

and the proof is complete. 
The following result then follows by the same method of proof as Corollary 5 of Montgomery-Smith [8] (cf. [6, Proposition 1.2.1]).

Corollary 3. Fix $\rho \in(0,1)$. Let $X_{1}, \ldots, X_{n}$ be an exchangeable sequence of random variables with $\rho n \geq 1$. Let $m=\lfloor\rho n\rfloor$. Let $\alpha_{1}, \ldots, \alpha_{n}$ be an arbitary sequence of complex constants with $\left|\alpha_{i}\right| \leq 1$ for all $i$. Then,

$$
P\left(\left\|\sum_{i=1}^{n} \alpha_{i} X_{i}\right\|>\lambda\right) \leq c P\left(\left\|\sum_{i=1}^{m} X_{i}\right\|>\lambda / c\right),
$$

for all $\lambda \geq 0$, where $c=c(\rho) \in(0, \infty)$ is an absolute constant depending only on $\rho$.

We also obtain the following comparison inequality for tail probabilities of random sums of arbitrary random variables.

Corollary 4. Fix $\rho \in(0,1)$. Let $X_{1}, \ldots, X_{n}$ be arbitrary random variables. Assume that $\rho n \geq 1$. Put $m=\lfloor\rho n\rfloor$. For $1 \leq k \leq n$, let $A_{k}$ be a random subset of $[n]$ with cardinality $k$, where all such subsets have equal probability $1 /\left(\begin{array}{l}n \\ k\end{array}\right)$, and where the $A_{k}$ are independent of the $X_{i}$. Then,

$$
P\left(\left\|\sum_{i \in A_{k}} X_{i}\right\|>\lambda\right) \leq c P\left(\left\|\sum_{i \in A_{m}} X_{i}\right\|>\lambda / c\right),
$$

whenever $1 \leq k \leq n$ and $\lambda \geq 0$, where $c=c(\rho) \in(0, \infty)$ is a constant depending only on $\rho$. Moreover,

$$
P\left(\left\|\sum_{i \in A_{j}} X_{i}\right\|>\lambda\right) \leq c^{\prime} P\left(\left\|\sum_{i \in A_{k}} X_{i}\right\|>\lambda / c^{\prime}\right),
$$

whenever $1 \leq j \leq k \leq m$ and $\lambda \geq 0$, where $c^{\prime}=c^{\prime}(\rho) \in(0, \infty)$ is a constant depending only on $\rho$.

Proof. Let $\tau$ be a random permutation of $[n]$, with all $n$ ! such permutations having equal probability and with $\tau$ independent of the $X_{i}$. Let $Y_{i}=X_{\tau(i)}$. Then, $Y_{1}, \ldots, Y_{n}$ are exchangeable. Moreover, $\sum_{i \in A_{j}} X_{i}$ has the same probability distribution as $\sum_{i=1}^{j} Y_{i}$, so that (5) and (6) follow from Corollary 2 and Theorem 1 (with $\gamma=1 / \rho)$, respectively.

Remark 1. An examination of our proofs will show that the constant $c=c(\gamma)$ in Theorem 1 may be taken to be

$$
c(\gamma)=C_{1} \frac{\gamma^{2}}{(\gamma-1)^{2}}
$$

where $C_{1}$ is an absolute constant. Likewise, it can be seen by analyzing our proofs that the constant $c=c(\rho)$ in Corollaries 2 and 3 can be taken to be

$$
c(\rho)=C_{2} \frac{1}{\rho(1-\rho)^{2}},
$$

where $C_{2}$ is an absolute constant. The constant in (5) can be taken to be the constant of Corollary 2, while the constant in (6) can be chosen to just be the constant of Theorem 1 with $\gamma=\rho^{-1}$. It is not known whether the degrees of dependency on $\gamma$ or $\rho$ in any of the above constants can be improved. 
Note also that the constant $c$ appeared twice in (3). We could, thus, rewrite (3) with $c_{1}(\gamma)$ in place of the first occurrence of $c$ in $(3)$ and $c_{2}(\gamma)$ in place of the second. Our proofs then yield the validity of the rewritten (3) with

$$
c_{1}(\gamma)=\frac{15 \bar{\gamma}^{2}+3 \bar{\gamma}}{(\bar{\gamma}-1)^{2}}
$$

and

$$
c_{2}(\gamma)=\frac{24 \bar{\gamma}^{2}}{(\bar{\gamma}-1)^{2}}
$$

where $\bar{\gamma}=\min (\gamma, 2)$. It is most unlikely that the numerical values here are optimal, and it is not known whether the degrees of $\gamma$-dependence are optimal in either $c_{1}$ or $c_{2}$.

We now proceed to the proof of Theorem 1. First we need a certain definition of Montgomery-Smith [8]. We say that $x$ is a $\lambda$-concentration point for a random variable $X$ if $P(\|X-x\| \leq \lambda) \geq \frac{2}{3}$. The following result is crucial.

Proposition 1 (Montgomery-Smith [8]). Let $X_{1}, \ldots, X_{n}$ be exchangeable random variables. Fix $1 \leq k \leq n$, and suppose that the partial sums $S_{j}=\sum_{i=1}^{j} X_{i}$ have $\lambda$-concentration points $s_{j}$ whenever $1 \leq j \leq k$. Then $\left\|k s_{j}-j s_{k}\right\| \leq 3(k+j) \lambda$ whenever $1 \leq j \leq k$.

Montgomery-Smith [8] states this in the independent and identically distributed case. However, the proof in the exchangeable case is identical.

A random variable $U$ is said to be symmetric if $U$ and $-U$ have the same distribution. We say that a sequence $U_{1}, \ldots, U_{n}$ of random variables is jointly symmetric if $\left(U_{1}, \ldots, U_{n}\right)$ has the same joint distribution as $\left(\varepsilon_{1} U_{1}, \ldots, \varepsilon_{n} U_{n}\right)$ for any choice of signs $\varepsilon_{i} \in\{-1,1\}$. While Lévy's inequality is normally stated for sums of independent Banach space valued symmetric random variables, the same standard proof works just as well for jointly symmetric Banach space valued sequences of random variables and shows that if $U_{1}, \ldots, U_{n}$ are jointly symmetric, then

$$
P\left(\left\|\sup _{1 \leq j \leq n} \sum_{i=1}^{j} U_{i}\right\|>\lambda\right) \leq 2 P\left(\left\|\sum_{i=1}^{n} U_{i}\right\|>\lambda\right),
$$

for every $\lambda \geq 0$.

Recall that we have defined $[n]=\{1, \ldots, n\}$ for any positive integer $n$.

Lemma 1. Let $X_{1}, \ldots, X_{n}, Y_{1}, \ldots, Y_{n}$ be a sequence of exchangeable Banach space valued random variables. Put $S_{k}=\sum_{i=1}^{k} X_{i}$ and $T_{k}=\sum_{i=1}^{k} Y_{i}$. Then for any $\lambda \geq 0$ we have

$$
P\left(\sup _{1 \leq k \leq n}\left\|S_{k}-T_{k}\right\|>\lambda\right) \leq 2 P\left(\left\|S_{n}-T_{n}\right\|>\lambda\right)
$$

Proof. Let $U_{i}=X_{i}-Y_{i}$. Exchangeability then shows that $\left(X_{i}, Y_{i}\right)$ and $\left(Y_{i}, X_{i}\right)$ have the same conditional distributions given the $\sigma$-field

$$
\mathcal{F}_{i} \stackrel{\text { def }}{=} \sigma\left(U_{1}, \ldots, U_{i-1}, U_{i+1}, \ldots, U_{n}\right)
$$

for any fixed $i \in[n]$. Therefore, $U_{i}$ and $-U_{i}$ have the same conditional distributions given $\mathcal{F}_{i}$, and it follows that $U_{1}, \ldots, U_{n}$ are jointly symmetric. The desired inequality then follows immediately from Lévy's inequality (7). 
Lemma 2. Fix $\gamma \in(1,2]$. Let $n^{\prime}=\lceil\gamma n\rceil$. Suppose that $X_{1}, \ldots, X_{n^{\prime}}$ are exchangeable Banach space valued random variables. Then,

$$
P\left(\sup _{1 \leq i \leq n^{\prime}-n}\left\|\sum_{i=1}^{j} X_{i}\right\|>\lambda\right) \leq c P\left(\left\|\sum_{i=1}^{n} X_{i}\right\|>\lambda / c\right),
$$

for all $\lambda \geq 0$, where $c=c(\gamma) \in(0, \infty)$ depends only on $\gamma$.

If $\gamma=2$, Theorem 1 is obviously equivalent to Lemma 2. For other values of $\gamma$, we need the simple argument below, which is the same argument as was used to show that Theorem 1 implies Corollary 2.

Proof of Theorem 1. Replacing $\gamma$ by $\min (\gamma, 2)$, we may assume that $\gamma \in(1,2]$. Without loss of generality, $n^{\prime}=\lceil\gamma n\rceil$. Put $S_{j}=\sum_{i=1}^{j} X_{i}$. Let $r=n^{\prime}-n$. Let $N=\lceil n / r\rceil$. Note that $r \geq(\gamma-1) n$ so that $N \leq 1+(\gamma-1)^{-1}$. Fix $\lambda \geq 0$. Let

$$
E_{k}=\left\{\sup _{1 \leq j \leq \max ((k+1) r, n)-k r}\left\|\sum_{i=k r+1}^{k r+j} X_{i}\right\|>\frac{\lambda}{N}\right\},
$$

for $0 \leq k<N$. By exchangeability and Lemma 2 , we have

$$
\begin{aligned}
P\left(E_{k}\right) & =P\left(\sup _{1 \leq j \leq \max ((k+1) r, n)-k r}\left\|\sum_{i=1}^{j} X_{i}\right\|>\frac{\lambda}{N}\right) \\
& \leq c P\left(\left\|S_{n}\right\|>\frac{\lambda}{N c}\right),
\end{aligned}
$$

where $c$ depends only on $\gamma$. It is easy to see that if $\omega \notin \bigcup_{i=0}^{N-1} E_{k}$, then $\left\|S_{j}(\omega)\right\| \leq \lambda$ for all $j \in[n]$. Thus by (8) we have

$$
\begin{aligned}
P\left(\sup _{1 \leq j \leq n}\left\|S_{j}\right\|>\lambda\right) & \leq P\left(\bigcup_{k=0}^{N-1} E_{k}\right) \\
& \leq N c P\left(\left\|\sum_{i=1}^{n} X_{i}\right\|>\frac{\lambda}{N c}\right) \\
& \leq\left(1+(\gamma-1)^{-1}\right) c P\left(\left\|\sum_{i=1}^{n} X_{i}\right\|>\frac{\lambda}{\left(1+(\gamma-1)^{-1}\right) c}\right) .
\end{aligned}
$$

It remains to prove Lemma 2. The careful reader will note that the proof in the case $\gamma=2$ simplifies somewhat.

Proof of Lemma 2. Let $r=n^{\prime}-n$. Note that $r \leq n$ as $\gamma \leq 2$. Put $S_{j}=\sum_{i=1}^{j} X_{i}$ for $j \in[n]$. Set $Y_{i}=X_{n+i}$ for $i \in[r]$, and define $T_{j}=\sum_{i=1}^{j} Y_{i}$. Fix $\lambda \geq 0$. Let

$$
p=P\left(\left\|S_{n}\right\|>\lambda\right) \text {. }
$$

Note that $S_{n}=S_{r}+\left(S_{n}-S_{r}\right)$ and that exchangeability then shows that $S_{n}$ has the same distribution as $T_{r}+\left(S_{n}-S_{r}\right)$. Hence we have

$$
\begin{aligned}
& P\left(\left\|S_{r}-T_{r}\right\|>2 \lambda\right) \\
& \quad=P\left(\left\|S_{r}+\left(S_{n}-S_{r}\right)-\left[T_{r}+\left(S_{n}-S_{r}\right)\right]\right\|>2 \lambda\right) \\
& \quad \leq P\left(\left\|S_{n}\right\|>\lambda\right)+P\left(\left\|T_{r}+\left(S_{n}-S_{r}\right)\right\|>\lambda\right)=2 p .
\end{aligned}
$$


By Lemma 1 we then have

$$
P\left(\sup _{1 \leq j \leq r}\left\|S_{j}-T_{j}\right\|>2 \lambda\right) \leq 4 p .
$$

Let $N=\lceil n / r\rceil$. We have $N \geq 1$ since $r \leq n$. Note that $r \geq(\gamma-1) n$ so that $N \leq 1+(\gamma-1)^{-1}$. For $0 \leq k<N$ and $r k<j \leq \min (r(k+1), n)$, let

$$
T_{j}^{\prime}=k T_{r}+T_{j-r k} .
$$

Note that $T_{r k}^{\prime}=k T_{r}$. Define $T_{0}^{\prime}=S_{0}=0$. Suppose that $r k<j \leq \min (r(k+1), n)$. Then,

$$
\begin{aligned}
\left\|S_{j}-T_{j}^{\prime}\right\| & =\left\|\sum_{l=1}^{k}\left(S_{r l}-S_{r(l-1)}-T_{r}\right)+\sum_{i=r k+1}^{j}\left(X_{i}-Y_{i-r k}\right)\right\| \\
& \leq \sum_{l=1}^{N}\left\|S_{r l}-S_{r(l-1)}-T_{r}\right\|+\left\|\sum_{i=r k+1}^{j}\left(X_{i}-Y_{i-r k}\right)\right\| .
\end{aligned}
$$

Let $F_{1}=\left\{\sup _{1 \leq j \leq n}\left\|S_{j}-T_{j}^{\prime}\right\|>4 N \lambda\right\}$. Because of (12) we then have $P\left(F_{1}\right) \leq$ $q_{1}+q_{2}$, where

$$
\begin{aligned}
q_{1} & =P\left(\sum_{l=1}^{N-1}\left\|S_{r l}-S_{r(l-1)}-T_{r}\right\|>2 N \lambda\right) \\
& \leq \sum_{l=1}^{N-1} P\left(\left\|S_{r l}-S_{r(l-1)}-T_{r}\right\|>2 \lambda\right) \\
& =\sum_{l=1}^{N-1} P\left(\left\|S_{r}-T_{r}\right\|>2 \lambda\right) \\
& \leq 2(N-1) p
\end{aligned}
$$

by exchangeability and (10), and where

$$
\begin{aligned}
q_{2} & \leq P\left(\sup _{\substack{0 \leq k<N \\
r k<j \leq \min (r(k+1), n)}}\left\|\sum_{i=r k+1}^{j}\left(X_{i}-Y_{i-r k}\right)\right\|>2 N \lambda\right) \\
& \leq \sum_{k=0}^{N-1} P\left(\sup _{r k<j \leq \min (r(k+1), n)}\left\|\sum_{i=r k+1}^{j}\left(X_{i}-Y_{i-r k}\right)\right\|>2 N \lambda\right) \\
& =\sum_{k=0}^{N-1} P\left(\sup _{1 \leq j \leq \min (r(k+1), n)-r k}\left\|\sum_{i=1}^{j}\left(X_{i}-Y_{i}\right)\right\|>2 N \lambda\right) \\
& \leq 4 N p,
\end{aligned}
$$

by exchangeability together with (11) and as $N \geq 1$. Hence, $P\left(F_{1}\right) \leq q_{1}+q_{2} \leq$ $(6 N-2) p$. Set

$$
F_{2}=\left\{\left\|S_{n}\right\|>\lambda\right\},
$$

and note that $P\left(F_{2}\right)=p$. Put $F=F_{1} \cup F_{2}$ so that we will have $P(F) \leq(6 N-1) p$. Let $\mathcal{G}=\sigma\left(Y_{1}, \ldots, Y_{r}\right)$. Let $E$ be the event $\left\{P(F \mid \mathcal{G}) \leq \frac{1}{3}\right\}$.

Then,

$$
(6 N-1) p \geq P(F)=E[P(F \mid \mathcal{G})] \geq \frac{1}{3}(1-P(E)),
$$


so that

$$
P(E) \geq 1-(18 N-3) p
$$

Note that $E \in \mathcal{G}$. But

$$
P\left(\sup _{1 \leq j \leq n}\left\|S_{j}-T_{j}^{\prime}\right\|>4 N \lambda \mid \mathcal{G}\right) \leq \frac{1}{3} \quad \text { on } E .
$$

(Note of course that $E$ could have measure zero, in which case this last assertion is trivial.) Hence, given $\mathcal{G}$ and $E$, the vector $T_{j}^{\prime}$ is a $4 N \lambda$-concentration point of $S_{i}$ whenever $1 \leq j \leq n$ since $P\left(F_{1} \mid \mathcal{G}\right) \leq \frac{1}{3}$ on $E$. Moreover, since $P\left(F_{2} \mid \mathcal{G}\right) \leq \frac{1}{3}$ on $E$, the vector 0 is a $\lambda$-concentration point of $S_{n}$ given $\mathcal{G}$ and $E$, and thus also a $4 N \lambda$-concentration point. Since the variables $X_{1}, \ldots, X_{n}$ can easily be seen to be (conditionally) exchangeable given $\mathcal{G}$, it follows from Proposition 1 that almost surely on $E$ we have

$$
\left\|j \cdot 0-n T_{j}^{\prime}\right\| \leq 12(n+j) N \lambda,
$$

for $1 \leq j \leq n$. But $T_{j}^{\prime}=T_{j}$ for $1 \leq j \leq r$. Moreover $(n+j) / n \leq 2$ for $1 \leq j \leq r$ since $(n+r) / n \leq 2$ as $\gamma \leq 2$, so that on $E$ we almost surely have $\left\|T_{j}\right\| \leq 24 N \lambda$ for $1 \leq j \leq r$. Therefore,

$$
\begin{aligned}
P\left(\sup _{1 \leq j \leq r}\left\|T_{j}\right\|>24 N \lambda\right) & \leq 1-P(E) \leq(18 N-3) p \\
& =(18 N-3) P\left(\left\|S_{n}\right\|>\lambda\right)
\end{aligned}
$$

by (13) and (9). By exchangeability, we may replace $T_{j}$ by $S_{j}$ on the left-hand side of this inequality and then since $\lambda \geq 0$ was arbitrary, it follows that

$$
\begin{aligned}
P\left(\sup _{1 \leq j \leq r}\left\|S_{j}\right\|>\lambda\right) & \leq(18 N-3) P\left(\left\|S_{n}\right\|>\lambda / 24 N\right) \\
& \leq\left(18(\gamma-1)^{-1}+15\right) P\left(\left\|S_{n}\right\|>\frac{\lambda}{24\left(1+(\gamma-1)^{-1}\right)}\right),
\end{aligned}
$$

as $N \leq 1+(\gamma-1)^{-1}$

\section{ACKNOWLEDGMENTS}

The author would like to thank Professor Stephen J. Montgomery-Smith for a number of interesting discussions. The author would also like to thank an anonymous referee for some helpful suggestions.

The research was partially supported by Professor J. J. F. Fournier's NSERC Grant \#4822 and was mostly done while the author was at the University of British Columbia.

\section{REFERENCES}

1. L. Crisma, Alcune valutazioni quantitative interessanti la proseguibilità di processi aleatori scambiabili, Rend. Ist. Mat. Univ. Trieste 3 (1971), 96-124. MR 45:7792

2. B. de Finetti, La prévision, ses lois logiques, ses sources subjectives, Ann. Inst. H. Poincaré 7 (1937), 1-68.

3. _ Sulla proseguibilità di processi aleatori scambiabili, Rend. Ist. Mat. Univ. Trieste 1 (1969), 53-67. MR 45:1233

4. Theory of probability, vols. 1 and 2, Wiley, New York, 1974-75. MR 55:13514a; MR 55:13514b

5. P. Diaconis, Finite forms of de Finetti's theorem on exchangeability, Synthese 36 (1977), 271-281. MR 58:24436 
6. Stanisław Kwapień and Wojbor A. Woyczyński, Random series and stochastic integrals: Single and multiple, Birkhauser, Boston, 1992. MR 94k:60074

7. R. Latała, On a maximal inequality for sums of independent identically distributed random variables, Warsaw University Preprint.

8. S. J. Montgomery-Smith, Comparison of sums of independent identically distributed random vectors, Probab. Math. Statist. 14 (1993), 281-285. MR 96b:60042

9. M. Scarsini, Lower bounds for the distribution function of a $k$-dimensional $n$-extendible exchangeable process, Statist. Probab. Lett. 3 (1985), 57-62. MR 86j:60040

10. F. Spizzichino, Extendibility of symmetric probability distributions and related bounds, Exchangeability in probability and statistics (G. Koch and F. Spizzichino, eds.), North-Holland, New York, 1982. MR 84g:60023

Department of Philosophy, University of Pittsburgh, Pittsburgh, Pennsylvania 15260

E-mail address: pruss+@pitt.edu 